\title{
Methylene Blue versus Coil-Based Computed Tomography-Guided Localization of Lung Nodules
}

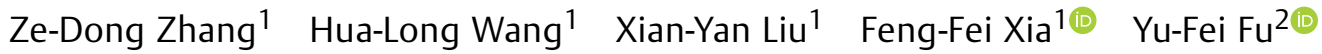 \\ ${ }^{1}$ Department of Interventional Vascular Surgery, Binzhou People's \\ Hospital, Binzhou, People's Republic of China \\ 2 Department of Radiology, Xuzhou Central Hospital, Xuzhou, \\ People's Republic of China \\ Address for correspondence Feng-Fei Xia, Binzhou People's Hospital, \\ 7 Huanghe Road, Binzhou 256600, People's Republic of China \\ (e-mail: xff510@126.com). \\ Thorac Cardiovasc Surg 2020;68:540-544.
}

\begin{abstract}
Background Preoperative computed tomography (CT)-guided localization has been shown to significantly improve lung nodule video-assisted thoracoscopic surgery (VATS)-based wedge resection technical success rates. However, at present, there was insufficient research regarding the optimal approaches to localization of these nodules prior to resection. We aimed to compare the relative clinical efficacy of preoperative CT-guided methylene blue and coil-based lung nodule localization.

Methods In total, 91 patients with lung nodules were subjected to either CT-guided methylene blue $(n=34)$ or coil $(n=57)$ localization and VATS resection from January 2014 to December 2018. We compared baseline data, localization-associated complication rates, as well as the technical success of localization and resection between these two groups of patients.

Results In total, 42 lung nodules in 34 patients underwent methylene blue localization, with associated localization and wedge resection technical success rates of 97.6 and $97.6 \%$, respectively. A total of 71 lung nodules in 57 patients underwent coil localization, with associated localization and wedge resection technical success rates of 94.4 and $97.2 \%$, respectively. There were no significant differences in technical success rates of localization or wedge resection between these groups $(p=0.416$ and 1.000 , respectively). The coil group sustained a longer duration between localization

\section{Keywords}

- localization

- methylene blue

- coil

- lung nodule and VATS relative to the methylene blue group (13.2 vs. 2.5 hours, $p=0.003$ ). Conclusion Both methylene blue and coil localization can be safely and effectively implemented for conducting the diagnostic wedge resection of lung nodules. The coilbased approach is compatible with a longer period of time between localization and VATS procedures.
\end{abstract}

\section{Introduction}

As low-dose computed tomography (CT) has increasingly been used for screening efforts, the rates of lung nodule detection have risen. ${ }^{1-3}$ To effectively determine whether the nodules are malignant or benign, wedge resection via video-assisted thoracoscopic surgery (VATS) has been widely used owing to its minimally invasive nature and the limited among or tissue resected during this procedure. ${ }^{4-6}$ The use of

received

November 26, 2019

accepted after revision

February 18, 2020

published online

April 20, 2020

preoperative CT-guided localization has been shown to significantly improve lung nodule VATS-based wedge resection technical success rates. ${ }^{7-9}$ However, at present, there has been insufficient research regarding the optimal approaches to localization of these nodules prior to resection. ${ }^{10,11}$

In the present study, we compared the relative clinical efficacy of preoperative CT-guided coil and methylene blue lung nodule localization strategies.

(c) 2020 Georg Thieme Verlag KG Stuttgart · New York
DOI https://doi.org/ 10.1055/s-0040-1708836. ISSN 0171-6425. 
Table 1 Comparison of data between coil and methylene blue groups

\begin{tabular}{|c|c|c|c|}
\hline & Coil group & $\begin{array}{l}\text { Methylene } \\
\text { blue group }\end{array}$ & $p$-Value \\
\hline \multicolumn{4}{|l|}{ Patient-based analysis } \\
\hline Patients number & 57 & 34 & \\
\hline Age (y) & $59.6 \pm 9.8$ & $60.1 \pm 11.0$ & 0.842 \\
\hline Gender (male/female) & $25 / 32$ & $13 / 21$ & 0.599 \\
\hline Tumor history & 15 & 5 & 0.196 \\
\hline $\begin{array}{l}\text { Patients with multiple } \\
\text { target nodules }\end{array}$ & 12 & 7 & 0.851 \\
\hline \multicolumn{4}{|l|}{ Nodule-based analysis } \\
\hline Nodules number & 71 & 42 & \\
\hline Diameter (mm) & $9.1 \pm 5.2$ & $9.7 \pm 4.2$ & 0.529 \\
\hline \multicolumn{4}{|l|}{ Natures of nodules } \\
\hline Solid & 33 & 24 & \multirow[t]{3}{*}{0.521} \\
\hline GGN & 11 & 6 & \\
\hline Mixed GGN & 27 & 12 & \\
\hline $\begin{array}{l}\text { Nodule-pleura } \\
\text { distance (mm) }\end{array}$ & $5.6 \pm 5.6$ & $8.4 \pm 7.1$ & 0.033 \\
\hline Side (left/right) & $25 / 46$ & $18 / 24$ & 0.419 \\
\hline $\begin{array}{l}\text { Lobe } \\
\text { (upper/nonupper) }\end{array}$ & $30 / 41$ & $20 / 22$ & 0.579 \\
\hline $\begin{array}{l}\text { Technical success } \\
\text { of localization }\end{array}$ & 67 & 41 & 0.416 \\
\hline $\begin{array}{l}\text { Successful wedge } \\
\text { resection }\end{array}$ & 69 & 41 & 1.000 \\
\hline $\begin{array}{l}\text { Duration of } \\
\text { localization (min) }\end{array}$ & $15.4 \pm 5.2$ & $14.3 \pm 3.6$ & 0.204 \\
\hline
\end{tabular}

Abbreviation: GGN, ground glass nodule.

\section{Materials and Methods}

This was a retrospective analysis conducted at a single center. Our Institutional Review Board approved this study, and the requirement for participant written consent was waived owing to the retrospective nature of the study design.

\section{Study Design}

A total of 91 patients underwent CT-guided localization and VATS resection of lung nodules at our center between January 2014 and December 2018 (-Table 1). Prior to December 2014, 34 patients had undergone methylene blue-based localization, whereas the remaining 57 patients treated after December 2014 underwent coil-based localization (-Fig. 1). Of these 91 patients, 19 had previously undergone respective surgery to remove tumors (lung cancer: 11; colorectal cancer: 4; liver cancer: 1 ; renal cancer: 1 ; esophageal cancer: 1 ; and breast cancer: 1 ). For each patient, discussions with thoracic surgeons, oncologists, and radiologists were used to inform decisions regarding the resection of lung nodules.

Study inclusion criteria included: (1) a maximal long-axis lesion diameter $\leq 30 \mathrm{~mm}$; (2) a nodule-pleura distance $\leq 30 \mathrm{~mm}$; and (3) nodules lacking a definite pathological diagnosis. Exclusion criteria included: (1) a lesion diameter $<3 \mathrm{~mm}$; (b) a typical benign lesion, such as a calcification or one that had reduced in size upon followup; and (3) diffused multiple lung nodules.

\section{CT-Guided Methylene Blue Localization}

A 16-row CT (Philips, Cleveland, Ohio, United States) device was used for all procedures, with respective tube voltage and

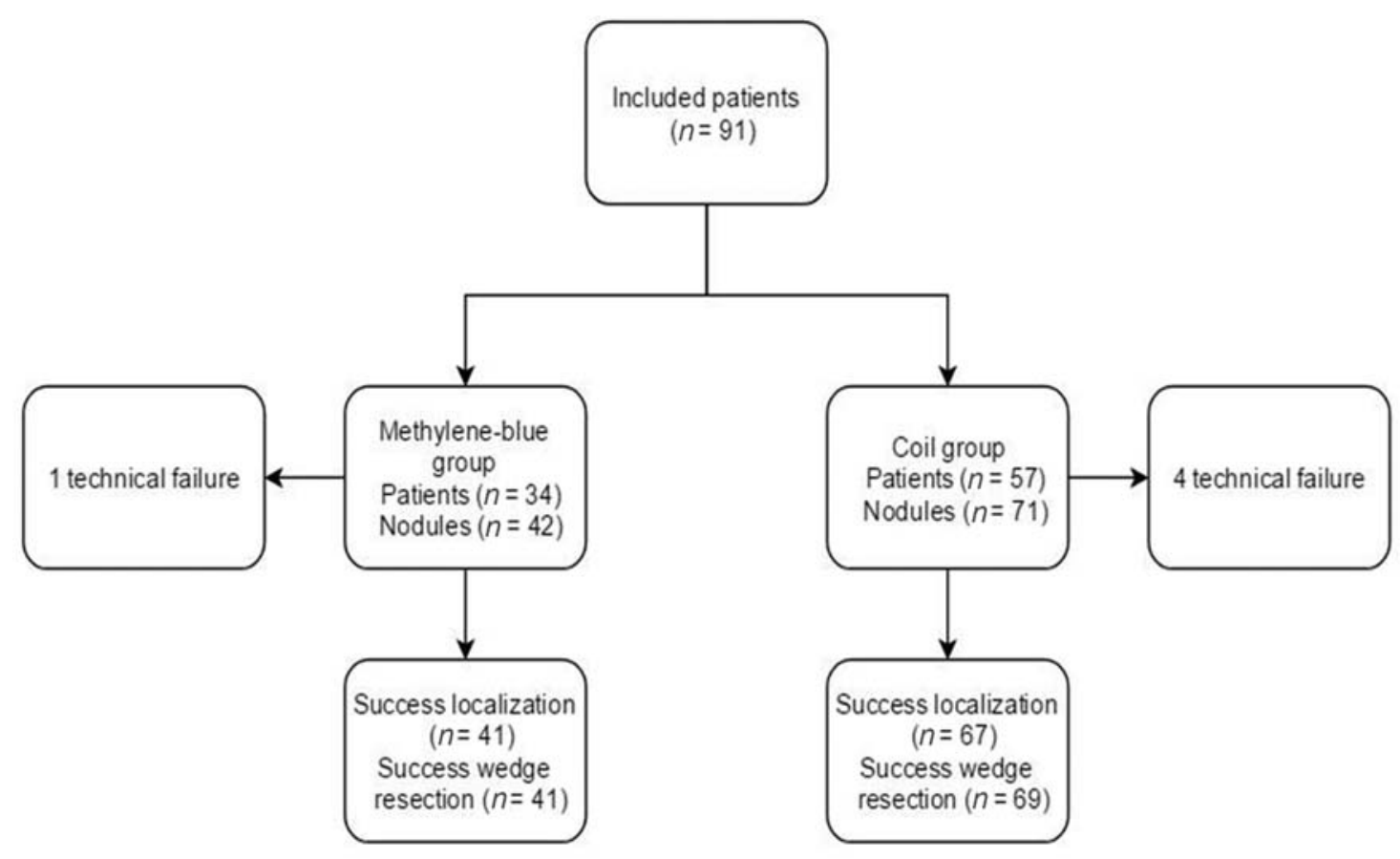

Fig. 1 The flowchart of this study. 


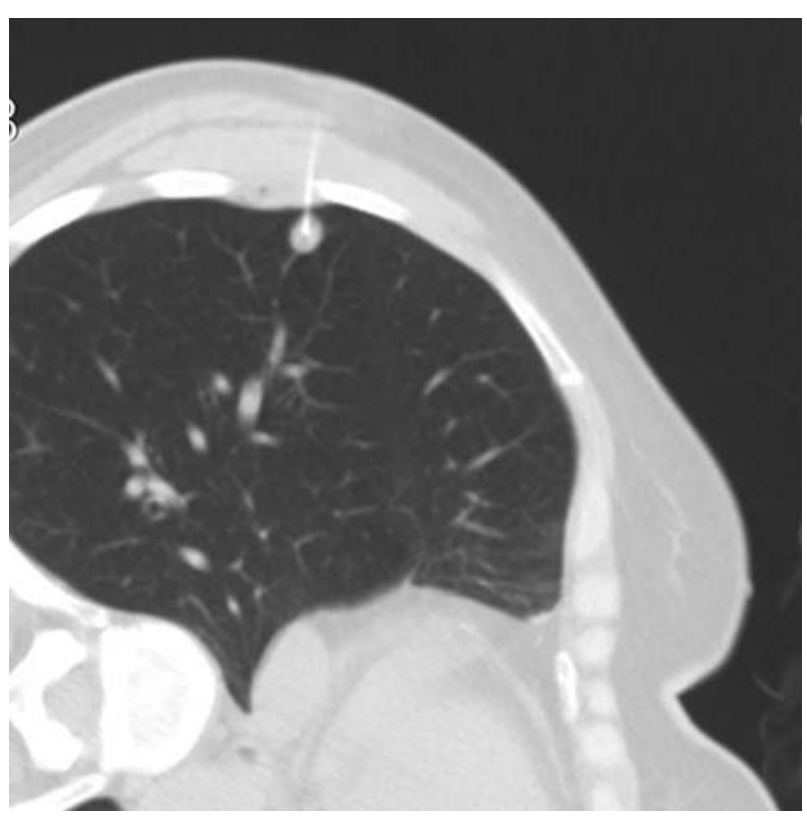

Fig. 2 The lung nodule was located by methylene blue which was injected by a $22 \mathrm{C}$ needle.

current settings of $120 \mathrm{kV}$ and $150 \mathrm{~mA}$ and a scanning thickness of $2 \mathrm{~mm}$.

Patients were positions so as to facilitate the shortest required pathway for needle insertion, after which the lung parenchyma was punctured using a 22G needle (Cook, Bloomington, Indiana, United States, -Fig. 2) positioned such that the needle tip was within $1 \mathrm{~cm}$ of the lesion site. Next, patients were steadily injected with $\sim 0.5 \mathrm{~mL}$ methylene blue while slowly removing the needle such that the methylene blue remained above the visceral pleura. A follow-up CT scan was then conducted to check for any procedure-associated complications.

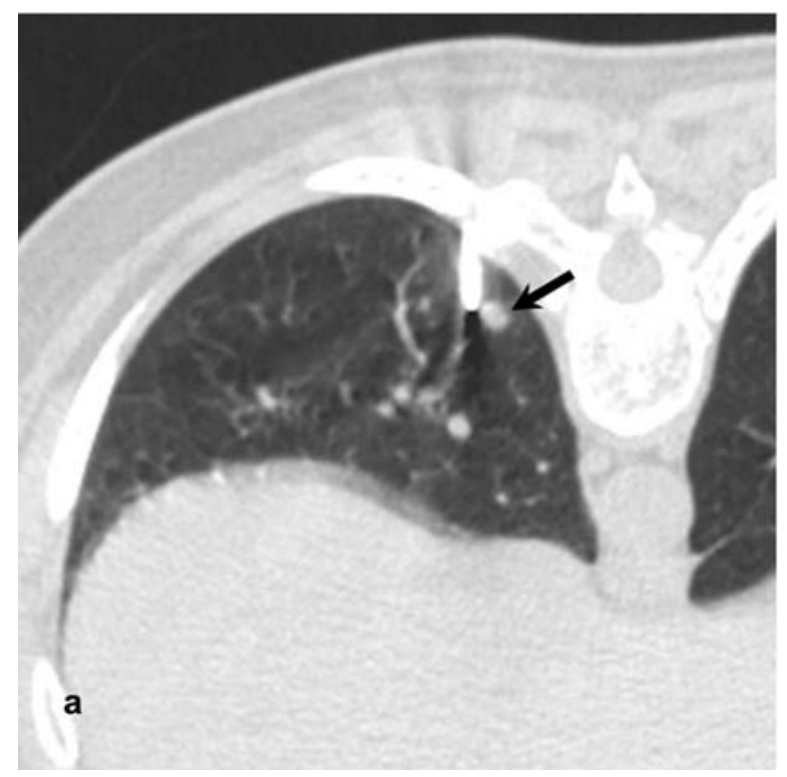

\section{CT-Guided Coil Localization}

All CT parameters were identical to those for the methylene blue localization group. Following needle insertion with an 18G needle (Precisa, Roma, Italy, - Fig. 3), a 50-mm long coil that was 0.038 inches in diameter (Cook) was partially inserted into the lung parenchyma. The needle was then gradually removed such that the tail of the coil remained above the visceral pleura. A follow-up CT scan was then conducted to check for any procedure-associated complications.

\section{VATS Procedure}

Owing to the risk of methylene blue diffusion, VATS in these patients was conducted within 3 hours of localization wherever possible. Wedge resection was conducted based on methylene blue visualization, and when such visualization was not possible, a lobectomy was instead conducted.

For patients who had undergone coil localization, VATS was performed within 24 hours of localization, with coil tail visualization being used to guide the wedge resection procedure. When the tail of the coil was not visible, palpation was used in an attempt to locate it. When this failed, a lobectomy was conducted.

The department of pathology conducted a rapid pathological examination of resected wedge lung parenchymal tissue. When nodules were diagnosed as being more advanced than mini-invasive adenocarcinoma, further lobectomy and lymph node dissection were conducted.

\section{Definitions}

The technical success of either CT-guided localization approach was based on the successful visualization of the methylene blue dye or the coil tail, as appropriate, when conducting the VATS procedure. Wedge resection technical success was defined based up the identification of the lesion of interest within the resected lung parenchymal tissue.

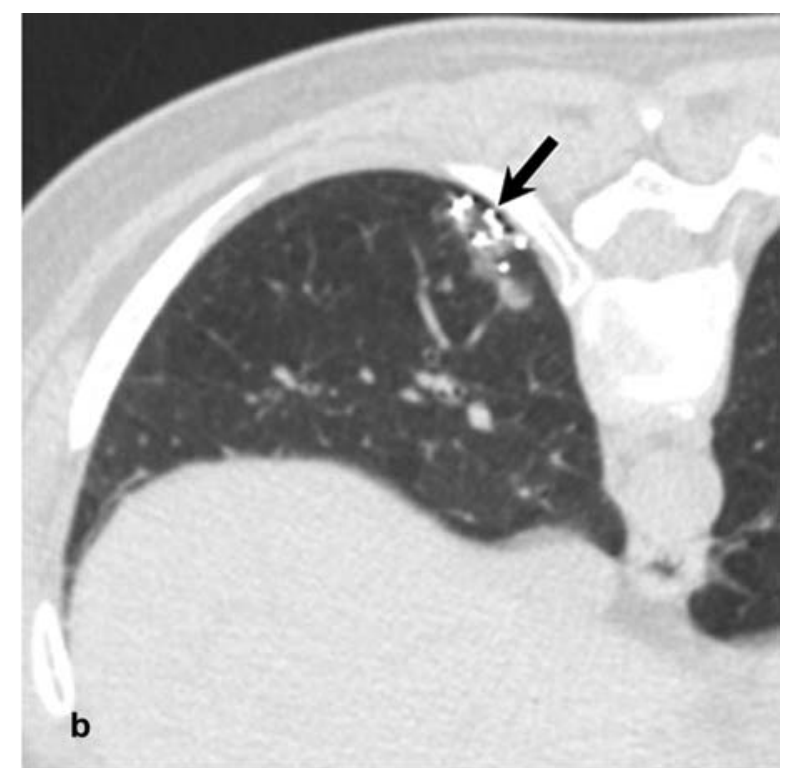

Fig. 3 The lung nodule was located by coil which was sent by an $18 \mathrm{G}$ needle. (A) The needle was sent near the nodule (arrow). (B) The coil (arrow) was partially placed in the lung tissue and the coil tail remained above the visceral pleura. 


\section{Statistical Analysis}

Categorical variables were compared via Fisher's exact test or chi-square tests. Continuous variables are means \pm standard deviation, and were compared via Student's $t$-tests or MannWhitney's $U$ tests. A $p<0.05$ was the significance threshold. SPSS v16.0 (SPSS Inc., Illinois, United States) was used for all statistical testing.

\section{Results}

\section{Technical Success of CT-Guided Localization}

A total of 42 lung nodules in 34 patients underwent methylene blue-based localization (-Table 1 ), with a technical success rate of $97.6 \%(41 / 42)$. Methylene blue was not detectable in the remaining patient owing to VATS having been conducted 24 hours postlocalization as a result of an unexpected rise in blood pressure.

A total of 71 lung nodules in 57 patients underwent coil localization ( - Table 1 ), with a technical success rate of $94.4 \%$ (67/71). The coil tail was not visible in the remaining four patients during VATS owing to the coil having been fully inserted into the parenchyma of the lung.

Localization technical success rates were comparable between these two study groups ( $p=0.416$ ).

\section{Procedure-Related Pneumothorax}

A total of three and seven patients in the methylene blue and coil groups, respectively, suffered from procedure-associated pneumothorax ( 8.8 vs. $12.3 \%, p=0.870$ ). Only a single patient in the coil group needed to undergo chest tube drainage, and this patient was still able to undergo VATS 24 hours following coil localization.

\section{Technical Success of Wedge Resection}

In the methylene blue and coil groups, the respective rates of wedge resection technical success were $97.6 \%(41 / 42)$ and $97.2 \%(69 / 71)(p=1.000,-$ Table 1$)$. Patients being assessed for multiple lung nodules underwent a single one-stage VATS procedure. Negative margins were confirmed in all resected segments of lung parenchymal tissue. While four patients in the coil localization group had suffered from technical failure during the localization procedure, two of these patients were able to undergo successful wedge resection because the tail of the coil was palpable during the VATS procedure.

Patients in the coil group had a significantly longer period of time between localization and VATS than those did in the methylene blue group (13.2 vs. 2.5 hours, $p=0.003$ ). However, there were no significant differences in VATS duration, blood loss, final diagnosis, or surgical types between these two localization groups (-Table 2 ).

\section{Discussion}

In this study, we compared the relative clinical efficacy of methylene blue and coil-based approaches to preoperatively localize lung nodules. We found that the rates of technical success for both localization (97.6 vs. 94.4, $p=0.416$ ) and subsequent wedge resection (97.6 vs. $97.2, p=1.000$ ) were comparable for these two approaches.

Many previous reports have highlighted the relative advantages of the two localization strategies utilized in the present study. ${ }^{4,10}$ Methylene blue localization offers the advantages of being both affordable and simple to implement, ${ }^{9}$ but its potential for rapid diffusion requires that VATS be conducted as quickly after localization as possible. Indeed, in the present

Table 2 Details of VATS and final diagnoses

\begin{tabular}{|c|c|c|c|}
\hline & Coil group & Methylene blue group & $p$-Value \\
\hline Nodules number & 71 & 42 & \\
\hline \multicolumn{4}{|l|}{ Types of surgery } \\
\hline Wedge resection & 49 & 32 & \multirow[t]{3}{*}{0.713} \\
\hline Wedge resection + lobectomy & 20 & 9 & \\
\hline Lobectomy & 2 & 1 & \\
\hline Duration between localization and VATS (h) & $13.2 \pm 25.5$ & $2.5 \pm 3.9$ & 0.003 \\
\hline Duration of VATS (min) & $151.5 \pm 76.3$ & $139.1 \pm 78.3$ & 0.458 \\
\hline Blood loss (mL) & $112.5 \pm 112.4$ & $103.2 \pm 98.3$ & 0.693 \\
\hline \multicolumn{4}{|l|}{ Final diagnoses } \\
\hline Invasive adenocarcinoma & 26 & 13 & \multirow[t]{7}{*}{0.975} \\
\hline MIS & 2 & 1 & \\
\hline AIS & 4 & 4 & \\
\hline Precancerous lesion & 10 & 5 & \\
\hline Squamous cells carcinoma & 1 & 1 & \\
\hline Metastasis & 1 & 1 & \\
\hline Benign & 27 & 17 & \\
\hline
\end{tabular}

Abbreviations: AIS, adenocarcinoma in situ; MIS, microinvasive adenocarcinoma; VATS, video-assisted thoracoscopic surgery. 
study, a single patient suffered from technical failure of the methylene blue-based localization approach owing to the delay which they experienced in undergoing VATS. While this only affected one patient in the present report, it underscores the importance of conducting VATS as quickly as possible. Consistent with this, we found that among assessed cases, there was a significantly longer period of time between localization and VATS for patients in the coil group relative to those in the methylene blue group $(p=0.003)$. This is consistent with the fact that coil localization can increase the window during which VATS can be conducted. However, coil localization is more complex to perform, necessitating increased operator skill levels.

Coil localization also offers an additional advantage over methylene blue localization, in that in some cases the coil may be inadvertently inserted fully into the parenchyma of the lung. In these cases, it may still be possible to conduct wedge resection provided the coil tail can be palpated during VATS. ${ }^{4}$ Indeed, in the present study, two of the four patients (50\%) who experienced coil localization technical failure were still successfully treated via VATS wedge resection owing to successful coil palpation.

We observed similar pneumothorax rates between the two study groups ( 8.8 vs. $12.3 \%, p=0.870$ ), suggesting these two localization strategies may have similar safety profiles. A previous study found the similar and high pneumothorax rates (46 vs. $50 \%$ ) after core (19G) and fine (22G) needle biopsy for lung nodules. ${ }^{12}$ Another study found the repeat punctures was the risk factor of the higher pneumothorax rate. ${ }^{13}$ Compared with CT-guided lung biopsy, CT-guided methylene blue or coil localization usually did not require repeat lung puncture. These findings may explain the reason why both needles ( $22 \mathrm{G}$ and $18 \mathrm{G}$ ) caused the low and similar pneumothorax rate in this present study. In addition, only a single patient in this study necessitates the insertion of a chest tube, suggesting pneumothorax does not have a significant impact on subsequent VATS performance.

Hookwire localization has been widely used to localize lung nodules in many previous reports, ${ }^{11,14}$ and while this procedure is simple to conduct, it suffers from substantially higher rates of complications. ${ }^{14}$ Previous studies ${ }^{11,14}$ comparing hookwire and coil localization complication rates found that the coilbased approach was associated with markedly reduced rates of patient pain and complications. Other researchers have employed the use of a radiolabel-based localization strategy, as this approach is also simple to implement and has a low risk of complications. ${ }^{15}$ This approach, however, necessitates the use of intraoperative X-ray guidance and exposes patients to significantly higher levels of radiation.

There are certain limitations to this study. First, this was a retrospective report and is thus susceptible to selective bias. Second, as the sample size was limited for this study, it is difficult to draw definitive conclusions regarding our findings. Third, this was a single-center study. As such, it is important that larger multicenter prospective studies be conducted in the future.

In summary, we found that both methylene blue and coilbased localization can be safely and effectively used to achieve high rates of successful lung nodule wedge resection for diagnostic purposes. Relative to methylene blue injection, the coil-based approach is compatible with a longer delay between the localization and VATS procedures.

\section{Funding}

None.

\section{Conflict of Interest}

None declared.

\section{Acknowledgments}

None.

\section{References}

1 Silva M, Pastorino U, Sverzellati N. Lung cancer screening with low-dose CT in Europe: strength and weakness of diverse independent screening trials. Clin Radiol 2017;72(05):389-400

2 Nair A, Hansell DM. European and North American lung cancer screening experience and implications for pulmonary nodule management. Eur Radiol 2011;21(12):2445-2454

3 Sverzellati N, Silva M, Calareso G, et al. Low-dose computed tomography for lung cancer screening: comparison of performance between annual and biennial screen. Eur Radiol 2016;26(11):3821-3829

4 Fu YF, Zhang M, Wu WB, Wang T. Coil localization-guided videoassisted thoracoscopic surgery for lung nodules. J Laparoendosc Adv Surg Tech A 2018;28(03):292-297

$5 \mathrm{Su}$ TH, Fan YF, Jin L, He W, Hu LB. CT-guided localization of small pulmonary nodules using adjacent microcoil implantation prior to video-assisted thoracoscopic surgical resection. Eur Radiol 2015;25 (09):2627-2633

6 Zhang M, Wang T, Wang H. A segmental labeling technique for nonintubated thoracoscopic anatomical segmentectomy. J Thorac Dis 2017;9(06):1648-1650

7 Finley RJ, Mayo JR, Grant K, et al. Preoperative computed tomography-guided microcoil localization of small peripheral pulmonary nodules: a prospective randomized controlled trial. J Thorac Cardiovasc Surg 2015;149(01):26-31

8 Lin MW, Chen JS. Image-guided techniques for localizing pulmonary nodules in thoracoscopic surgery.JThorac Dis 2016;8(Suppl9): S749-S755

9 Lin MW, Tseng YH, Lee YF, et al. Computed tomography-guided patent blue vital dye localization of pulmonary nodules in uniportal thoracoscopy. J Thorac Cardiovasc Surg 2016;152(02):535-544.e2

10 Kleedehn M, Kim DH, Lee FT, et al. Preoperative pulmonary nodule localization: a comparison of methylene blue and hookwire techniques. AJR Am J Roentgenol 2016;207(06):1334-1339

11 Hwang S, Kim TG, Song YG. Comparison of hook wire versus coil localization for video-assisted thoracoscopic surgery. Thorac Cancer 2018;9(03):384-389

12 Sangha BS, Hague CJ, Jessup J, O'Connor R, Mayo JR. Transthoracic computed tomography-guided lung nodule biopsy: comparison of core needle and fine needle aspiration techniques. Can Assoc Radiol J 2016;67(03):284-289

13 Yang W, Sun W, Li Q et al. Diagnostic accuracy of CT-guided transthoracic needle biopsy for solitary pulmonary nodules. PLoS One 2015;10(06):e0131373

14 Rostambeigi N, Scanlon P, Flanagan S, et al. CT fluoroscopic-guided coil localization of lung nodules prior to video-assisted thoracoscopic surgical resection reduces complications compared to hook wire localization. J Vasc Interv Radiol 2019;30(03):453-459

15 Sharma A, McDermott S, Mathisen DJ, Shepard JO. Preoperative localization of lung nodules with fiducial markers: feasibility and technical considerations. Ann Thorac Surg 2017;103(04):1114-1120 